\title{
System size, collision energy, and rapidity dependence of col- lective dynamics measured by the PHENIX experiment at RHIC
}

\author{
Seyoung $\operatorname{Han}^{1,2, \star}$ for the PHENIX collaborations \\ ${ }^{1}$ Ewha Womans University, Seoul, Republic of Korea \\ ${ }^{2}$ RIKEN, Saitama, Japan
}

\begin{abstract}
In high energy collisions, partons produced in the initial stage undergo multiple interactions and yield a collective motion as a whole. Recently, several questions have risen including how small the system can be for producing the collectivity, and how far in rapidity the collectivity extends. PHENIX has measured the particle flow in $p+\mathrm{Au}, d+\mathrm{Au}$, and ${ }^{3} \mathrm{He}+\mathrm{Au}$ collisions over several energies as well as over wider rapidity range. In this work, we summarize our results on the particle flow and discuss the implications for collectivity in small collision systems.
\end{abstract}

\section{Introduction}

Many studies of the properties of the Quark Gluon Plasma (QGP) have been done. One of the key observations in heavy-ion collisions is that the created medium behaves like almost ideal fluid; we call it the strongly coupled QGP. The collectivity in heavy ion systems is multi-particle correlations with the large rapidity gap which originated from a common source. On the other hand, there also exist non-collectivity originated correlation such as jet, parton decays for example. In the di-hadron correlations $v_{n}$, the coefficient of Fourier harmonics is commonly attributed to hydrodynamic flow.

Until recently, the collective behavior was not expected in small systems. The observation of a long-range, near-side ridge in high multiplicity $p+p$ by the CMS experiment challenged this view [1]. PHENIX has also studied the collectivity in small collision systems. Previously these studies have been only on the $p_{T}$ dependence in the most central events. More recent studies have expanded to all centralities to facilitate quantitative studies on the role of system size and non-flow effects, and also the rapidity $\eta$ dependence to study the longitudinal structure of the observed correlations.

\section{PHENIX detectors}

Table 1 shows data sets of small collision systems taken by the PHENIX experiment. We collected data from various small collision systems, namely $p+p, p+\mathrm{Au}, d+\mathrm{Au}$, and ${ }^{3} \mathrm{He}+\mathrm{Au}$ collisions at $\sqrt{s_{N N}}=200 \mathrm{GeV}$. Especially for $d+$ Au collisions in 2016, we also have beam energy scan data in four collision energies [2]. With these data sets, we can study a variety of observables, such as the system size dependence and collision geometry.

^e-mail: seyoung_han@ewhain.net 
Table 1. Small system data taken by PHENIX experiment.

\begin{tabular}{lllll}
\hline & ${ }^{3} \mathrm{He}+\mathrm{Au}$ & $d+\mathrm{Au}$ & $p+\mathrm{Au}$ & $p+p$ \\
\hline $200 \mathrm{GeV}$ & $\checkmark$ & $\checkmark$ & $\checkmark$ & $\checkmark$ \\
\hline $62.4 \mathrm{GeV}$ & & & & \\
\hline $39 \mathrm{GeV}$ & $\checkmark$ & & \\
\hline $19.6 \mathrm{GeV}$ & $\checkmark$ & & \\
\hline
\end{tabular}

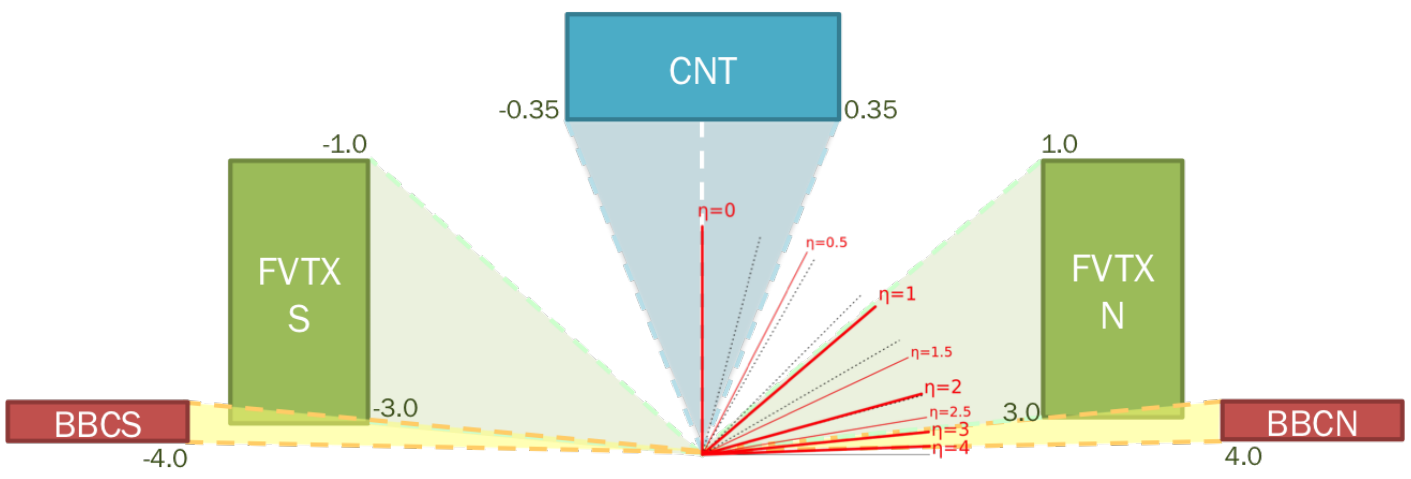

Figure 1. PHENIX detector coverage. The central arm (CNT) covers rapidity range of $-0.35<\eta<0.35$ with partial azimuthal coverage $(\Delta \phi=\pi)$. The FVTX (forward silicon vertex tracker) and BBC (beam beam counter) are implemented in both south and north directions and cover $1<|\eta|<3$ and $3<|\eta|<4$ with full azimuthal coverage.

Figure 1 shows the rapidity coverage of a part of the PHENIX detector subsystems involved in the present analysis. The central arm (CNT) which covers mid-rapidity can measure charged particles and particle identification with a limited acceptance is also possible. The FVTX and BBC are the spectrometers at forward (positive, north) and backward (negative, south) rapidity. In the collectivity study of small systems, we use the beam beam counter (BBC) for triggering minimum bias and high multiplicity events, calculating event plane resolutions, and measuring two particle correlations. We also use the forward silicon vertex tracker (FVTX) to measure charged tracks and event planes. In the $p+\mathrm{Au}, d+\mathrm{Au}$ and ${ }^{3} \mathrm{He}+\mathrm{Au}$ runs, particle multiplicity is higher at the south arm because Au ions move to the south arm, which is the backward rapidity. Therefore, the BBC and FVTX located at backward rapidity are used for event plane determination.

The PHENIX detectors have an advantage in wide acceptance range which allows us to study correlations in various pseudo-rapidity $(\eta)$ ranges. Figure 2 shows the two particle correlation functions of different $\Delta \eta$ gaps in $0-5 \%$ central $d+$ Au collisions at $\sqrt{s_{N N}}=200 \mathrm{GeV}$ [2]. $\Delta \eta$ gap increases from left to right. Since clear near-side ridge structure is shown in all combinations, we can expect non-zero $v_{2}$. In case of the correlation function between the central arm and the south FVTX, it shows the largest coefficient of the second order cosine modulation $\left(c_{2}\right)$. It is possible to include a larger effect from intra-jet or di-jet (non-flow effects) due to the small $\Delta \eta$ gap. As $\Delta \eta$ increases the non-flow effects are expected to be decreased and $c_{2}$ in Figure 2 shows a decreasing trend. However, we can still see a near-side ridge structure even in the two particle correlation function in $6.2<\Delta \eta<7.8$, where the non-flow effects are expected to be negligible. 

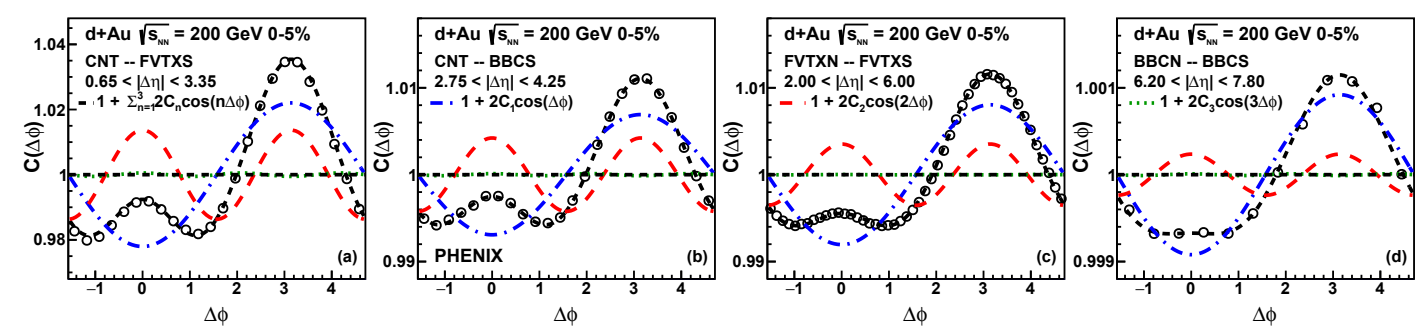

Figure 2. Two particle correlation functions in various $\Delta \eta$ gaps in $d+$ Au collisions at $\sqrt{s_{N N}}=200 \mathrm{GeV}[2]$.

\section{Analysis method}

Two independent analysis methods are used for the collectivity study in small systems. One method is using event plane which is measured by the south FVTX or the south BBC.

The event plane resolution is calculated by comparing event planes from the three independent systems. We used the central arm, the south FVTX, and the south BBC for the resolution calculation and applied to the raw $v_{2}$. The raw $v_{2}$ of charged particles at the mid-rapidity is the amplitude of $\cos 2 \phi$ modulation from the $\Delta \phi$ distribution between the charged particles in the central arm and event plane.

The other method is two particle correlation method (2PC) [3] which uses the ratio between the angular correlation of the particle pairs in a same event and mixed event with normalized by the total number of pairs of each. The Fourier coefficients of the second order cosine modulation $\left(c_{2}\right)$ from the three detector combinations are used to get the $v_{2}$. We confirmed the consistency between these two methods. $p+$ Au centrality dependence with function of $p_{T}$ is the result by using the two particle correlation method.

\section{System size dependence of $v_{2}$ and $v_{3}$}
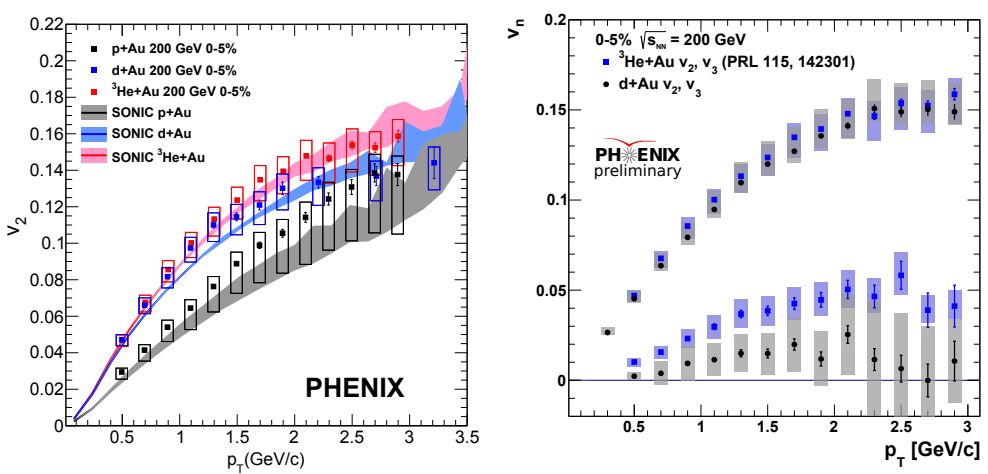

Figure 3. Collectivity measurement of charged hadrons at mid-rapidity as a function of $p_{T}$ in $0-5 \%$ central events. (left) $v_{2}$ of $p+\mathrm{Au}, d+\mathrm{Au}$, and ${ }^{3} \mathrm{He}+\mathrm{Au}$ collisions at $\sqrt{s_{N N}}=200 \mathrm{GeV}$. (right) $v_{2}$ and $v_{3}$ of $d+\mathrm{Au}$ and ${ }^{3} \mathrm{He}+\mathrm{Au}$ collisions at $\sqrt{s_{N N}}=200 \mathrm{GeV}[4]$. 
PHENIX measured $v_{2}$ and $v_{3}$ of charged hadrons at the mid-rapidity as a function of $p_{T}$ in $0-5 \%$ central events $p+\mathrm{Au}$ [4], $d+\mathrm{Au}$ [2], and ${ }^{3} \mathrm{He}+\mathrm{Au}[5]$ collisions at $\sqrt{s_{N N}}=200 \mathrm{GeV}$. In case of the $v_{2}$ shown in Figure 3, sizable $v_{2}$ observed in all three collision systems, and the $v_{2}$ measured in $p+\mathrm{Au}$ collisions is slightly smaller than the $v_{2}$ measured in $d+\mathrm{Au}$ and ${ }^{3} \mathrm{He}+\mathrm{Au}$ collisions. The SONIC model [6] can describe the results in all three collision systems reasonably well.

The right plot of Figure 3 shows $v_{2}$ and $v_{3}$ of $0-5 \%$ of $d+\mathrm{Au}$ and ${ }^{3} \mathrm{He}+\mathrm{Au}$ collisions at $\sqrt{s_{N N}}=200 \mathrm{GeV}$. The measured $v_{2}$ in two collision systems are consistent, while the $v_{3}$ of ${ }^{3} \mathrm{He}+\mathrm{Au}$ is larger than the $v_{3}$ of $d+\mathrm{Au}$.

\section{Beam energy scan of $d+$ Au collisions}

\section{$5.1 v_{2}$ as a function of $p_{T}$ in various centrality bins}

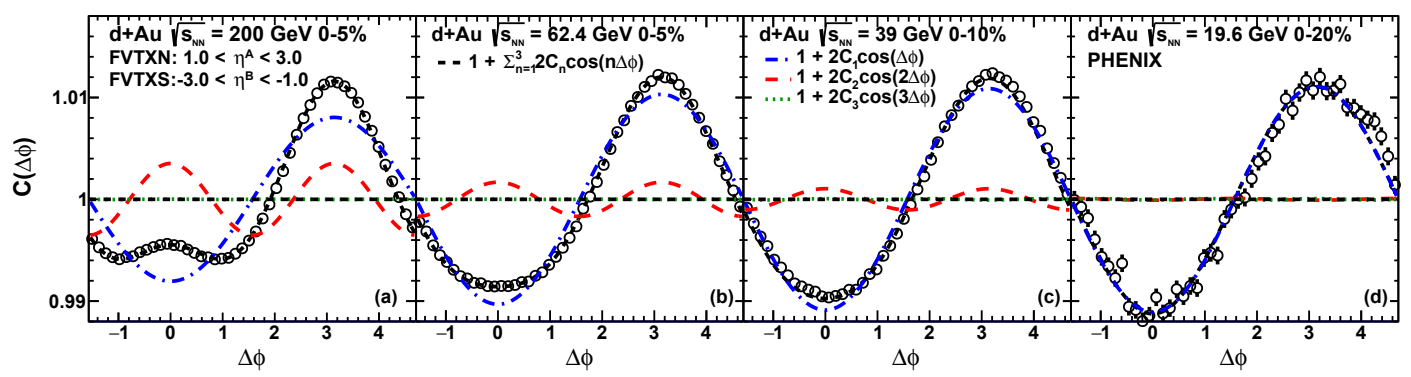

Figure 4. Two particle correlation between south and north FVTX in central $d+$ Au collisions at various collision energies [2].

Figure 4 shows two particle correlations of charged hadrons measured in the south and north FVTX in $d+\mathrm{Au}$ collisions at various collision energies [2]. As the collision energy decreases, $c_{2}$, which is the second order coefficient of cosine modulation (shown as red dashed lines) also decreases.

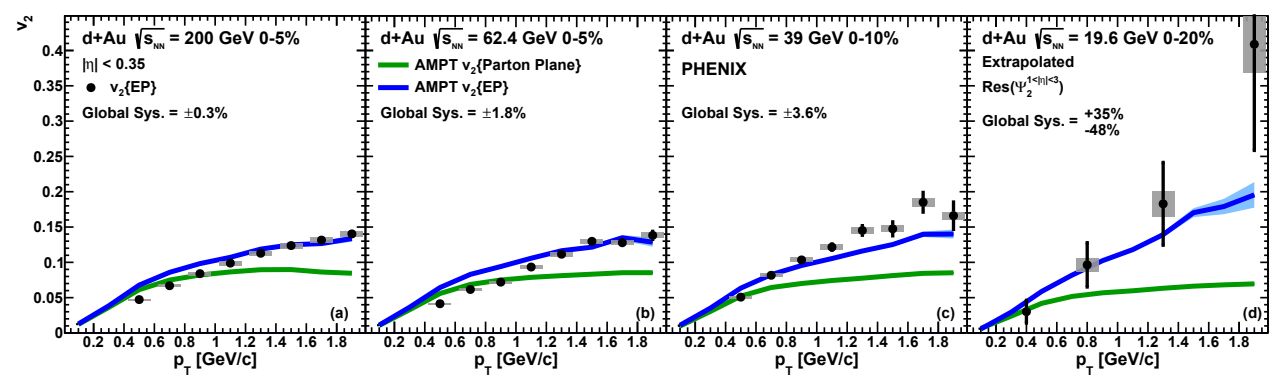

Figure 5. $v_{2}$ of charged hadrons as a function of $p_{T}$ at mid-rapidity in central $d+$ Au collisions at various collision energies.

For further understanding, the experimental data are compared with the AMPT [7] model $v_{2}$ calculations using both the parton plane and the event plane methods in Figure 5. The green curves $\left(v_{2}\{\right.$ Parton Plane $\left.\}\right)$ are the $v_{2}$ extracted by the parton plane method which is corresponding to initial 
collision geometry, and the blue curves $\left(v_{2}\{\mathrm{EP}\}\right)$ are measured by using relative angular position to the event plane based on the produced particles in Monte-Carlo simulation. The AMPT $v_{2}\{\mathrm{EP}\}$ calculations follow the real PHENIX detectors acceptance at the four collision energies. The results of the AMPT $v_{2}\{\mathrm{EP}\}$ follow the increasing trend of the $v_{2}$ data as collision energy becomes lower. On the other hand, in case of the AMPT $v_{2}$ \{Parton Plane\}, not only it is smaller than the data and the AMPT $v_{2}\{\mathrm{EP}\}$ but also it moderately decreases as the collision energy becomes lower. From these data comparisons with two sets of AMPT calculations, it is clear within the AMPT model that there are considerable fraction of the non-flow contribution in the measured $v_{2}$ especially dominated in the lower collision energy.

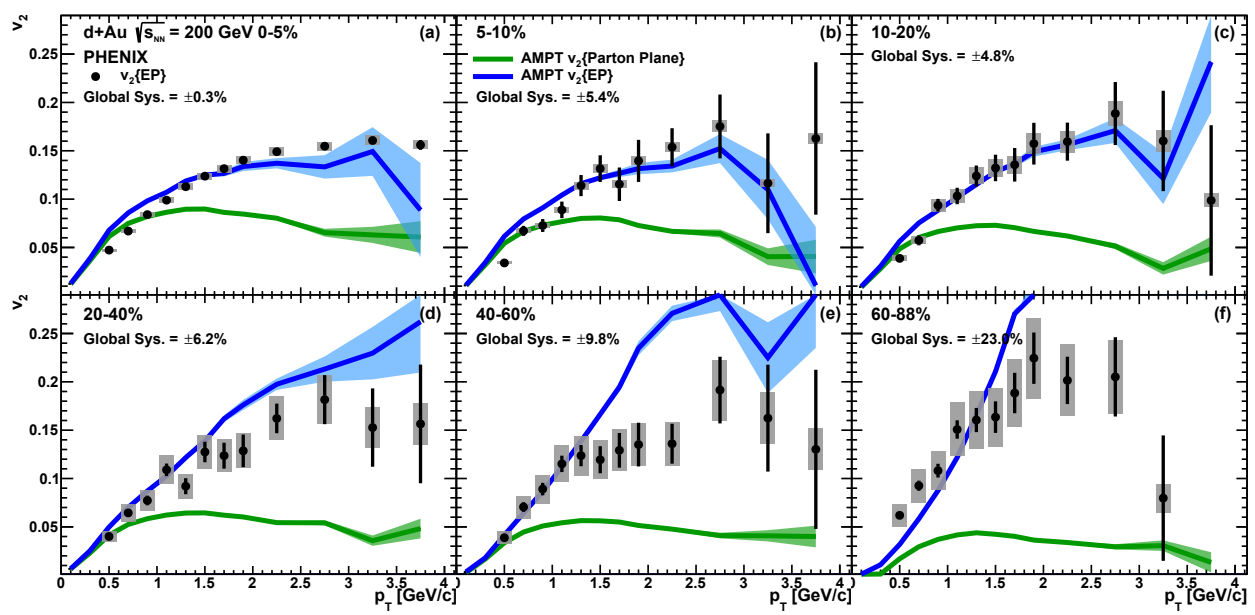

Figure 6. $v_{2}$ of charged hadrons as a function of $p_{T}$ at mid-rapidity in various centrality classes of $d+\mathrm{Au}$ collisions at $\sqrt{s_{N N}}=200 \mathrm{GeV}$.

Figure 6 shows $v_{2}$ of charged hadrons at mid-rapidity as a function of $p_{T}$ in a various centrality classes of $d+\mathrm{Au}$ collisions at $\sqrt{s_{N N}}=200 \mathrm{GeV}$. Non-zero $v_{2}$ is observed in all centrality bins, and measured value increases from central to peripheral collisions. The AMPT $v_{2}\{\mathrm{EP}\}$ agrees with the data in all centrality classes, and the AMPT $v_{2}$ \{Parton Plane $\}$ gives lower value than the data. In case of the AMPT $v_{2}\{$ Parton Plane $\}$, the amplitude decreases from central to peripheral collisions due to increasing non-flow contribution..

Figure 7 and 8 shows the results of $d+$ Au collisions at $\sqrt{s_{N N}}=62 \mathrm{GeV}$ and $39 \mathrm{GeV}$, respectively. From those two figures the amplitude of $v_{2}$ is slightly larger than that in the $\sqrt{s_{N N}}=200 \mathrm{GeV}$ when comparing the results in the same centrality bins. The AMPT $v_{2}\{\mathrm{EP}\}$ agrees with the data at $\sqrt{s_{N N}}=62 \mathrm{GeV}$ but gives lower value than the experimental data at $\sqrt{s_{N N}}=39 \mathrm{GeV}$ especially at the peripheral events. It is similar with the $\sqrt{s_{N N}}=200 \mathrm{GeV}$ results, the AMPT $v_{2}$ \{Parton Plane calculations always underestimate the data. The difference between the data and the AMPT $v_{2}$ \{Parton Plane $\}$ is larger in high $p_{T}$ and peripheral events due to a larger non-flow contribution.

In summary of the beam energy scan results, the measured $v_{2}$ is larger in higher $p_{T}$, peripheral collisions, and lower collision energy. The AMPT $v_{2}\{\mathrm{EP}\}$ reproduces general shape of $p_{T}$ dependence in $v_{2}$. On the contrary, the AMPT $v_{2}$ \{Parton Plane \} calculations excluding possible non-flow effects therefore tend to underestimate the experimental data. 


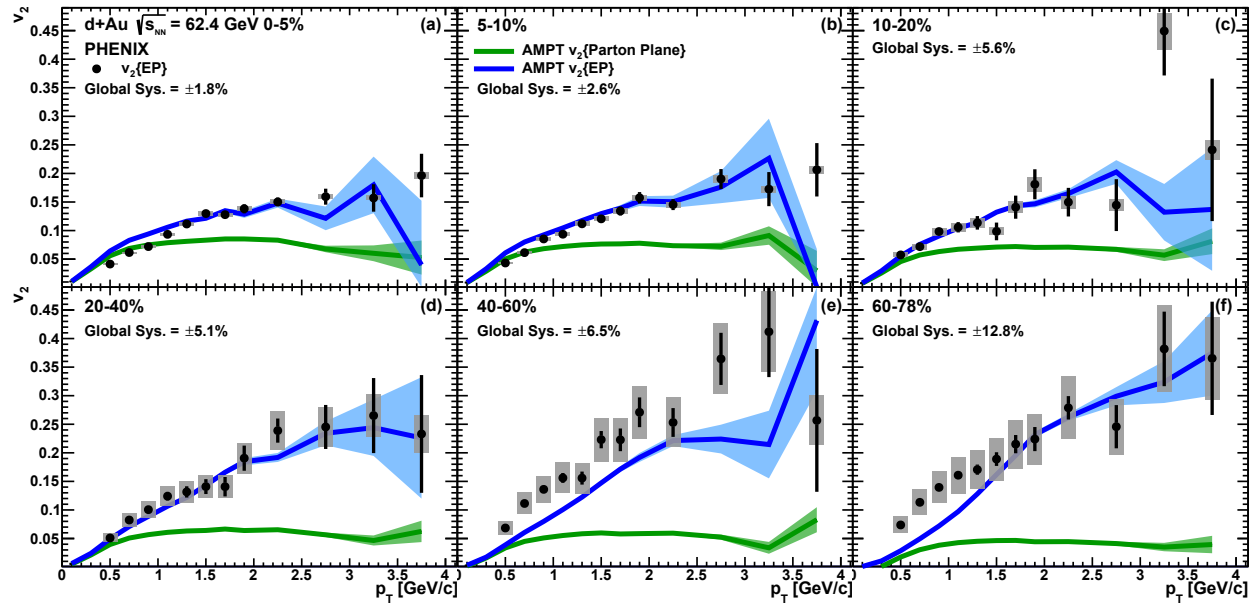

Figure 7. $v_{2}$ of charged hadrons as a function of $p_{T}$ at mid-rapidity in various centrality classes of $d+\mathrm{Au}$ collisions at $\sqrt{s_{N N}}=62 \mathrm{GeV}$.

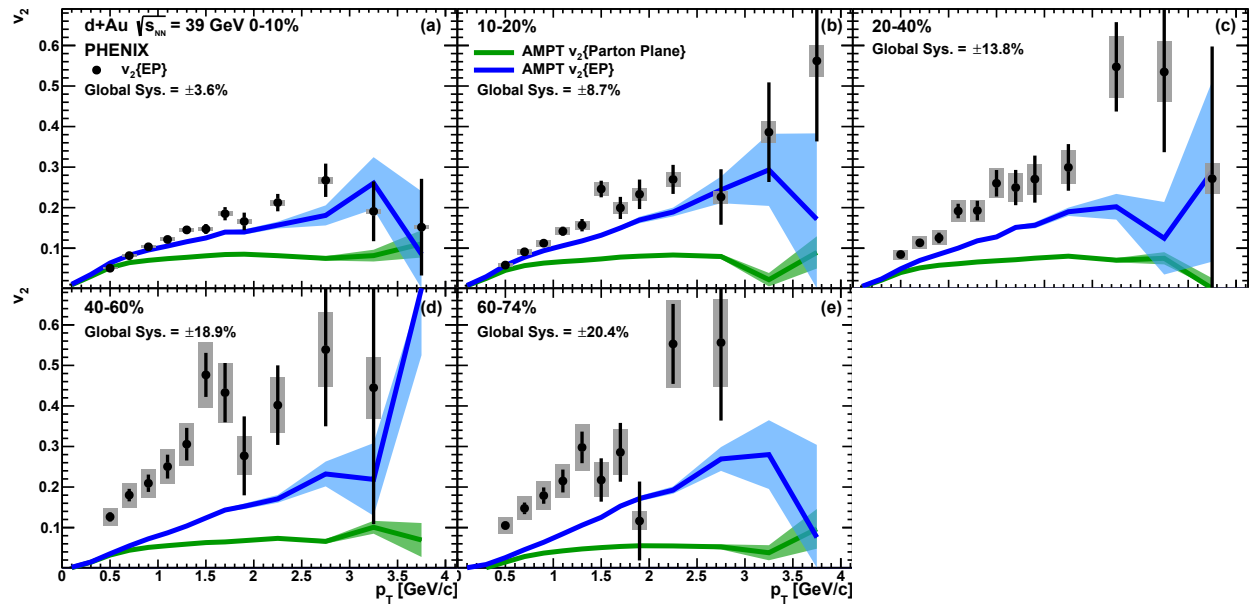

Figure 8. $v_{2}$ of charged hadrons as a function of $p_{T}$ at mid-rapidity in various centrality classes of $d+\mathrm{Au}$ collisions at $\sqrt{s_{N N}}=39 \mathrm{GeV}$.

\subsection{Rapidity dependence of $v_{2}$}

Figure 9 shows $v_{2}$ of charged hadrons as a function of $\eta$ in central $d+\mathrm{Au}$ collisions at $\sqrt{s_{N N}}=200 \mathrm{GeV}$ (left), $62 \mathrm{GeV}$ (middle), and $39 \mathrm{GeV}$ (right) comparisons with the AMPT simulation. There is a clear energy dependence at the backward rapidity, which is the direction of the $\mathrm{Au}$ beam, whereas the rapidity dependence is weaker at the forward rapidity. In addition, the $v_{2}$ of the backward rapidity is larger than that of the forward rapidity at high collision energy, but this asymmetric shape quenches as the collision energy becomes smaller. 


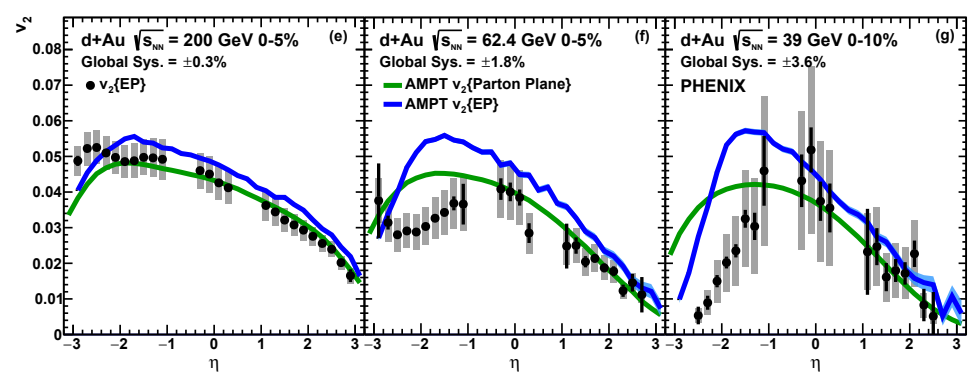

Figure 9. $v_{2}$ of charged hadrons as a function of $\eta$ in central $d+$ Au collisions at various collision energies and comparison with AMPT simulation.

The measured $v_{2}$ agrees with both AMPT $v_{2}\{$ EP $\}$ and AMPT $v_{2}\{$ Parton Plane $\}$ in all three collision energies at forward rapidity. Therefore at the backward rapidity the AMPT simulation reproduces the data at $\sqrt{s_{N N}}=200 \mathrm{GeV}$, but it overestimates $v_{2}$ at $\sqrt{s_{N N}}=62 \mathrm{GeV}$ and $39 \mathrm{GeV}$ while the AMPT $v_{2}\{\mathrm{EP}\}$ and $v_{2}$ \{Parton Plane $\}$ show a difference. This difference is possibly due to a larger non-flow contribution.

\section{$6 v_{2}$ function of $p_{T}$ in $p+$ Au collisions}

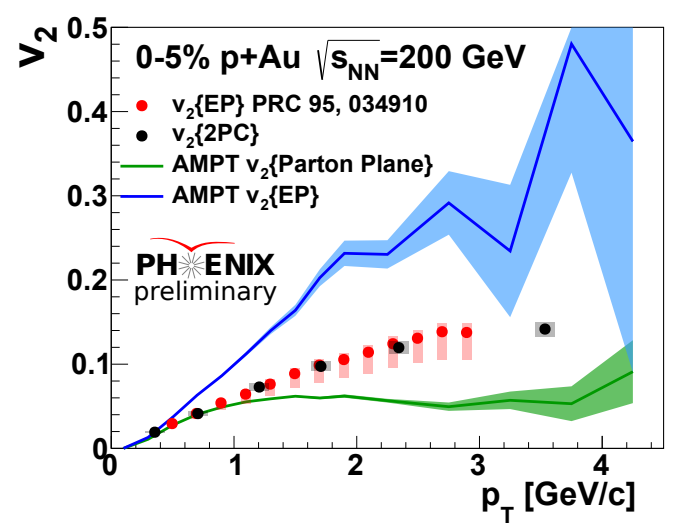

Figure 10. $v_{2}$ of charged hadrons as a function of $p_{T}$ in the $0-5 \%$ centrality for $p+$ Au collisions at $\sqrt{s_{N N}}=200 \mathrm{GeV}$ and comparison with AMPT simulation.

Figure 10 shows $v_{2}$ of charged hadrons as a function of $p_{T}$ at mid-rapidity in $0-5 \%$ central events in $p+\mathrm{Au} \sqrt{s_{N N}}=200 \mathrm{GeV}$ collisions. The new results of $v_{2}\{2 \mathrm{PC}\}$ with the three-sub detector method are consistent with the previous PHENIX results of $v_{2}\{\mathrm{EP}\}$. In comparison with the AMPT simulation, the measured $v_{2}$ agree with the AMPT $v_{2}$ \{Parton Plane $\}$ in $p_{T}<1 \mathrm{GeV} / c$, and the data in large $p_{T}$ are laid in between the AMPT $v_{2}\{\mathrm{EP}\}$ and $v_{2}\{$ Parton Plane $\}$. The tendency between the data and AMPT simulation in $p+$ Au collisions is different from that in the $d+$ Au collisions, which shows a good agreement between the data and the AMPT $v_{2}\{\mathrm{EP}\}$. In $p+$ Au collisions, AMPT calculations 


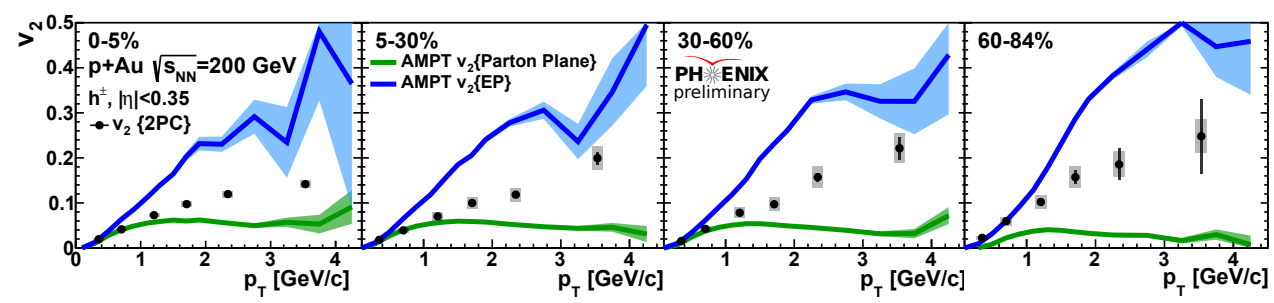

Figure 11. $v_{2}$ of charged hadrons as a function of $p_{T}$ in various centrality classes of $p+\mathrm{Au}$ collisions at $\sqrt{s_{N N}}=200 \mathrm{GeV}$ and comparison with AMPT simulation.

overestimate $v_{2}\{\mathrm{EP}\}$ in all centralities. Figure 11 shows $v_{2}\{2 \mathrm{PC}\}$ of charged hadrons as a function of $p_{T}$ at mid-rapidity in various centrality classes of $p+$ Au collisions at $\sqrt{s_{N N}}=200 \mathrm{GeV}$. The amplitude of $v_{2}$ in $p+$ Au collisions is similar with the results in $d+$ Au collisions, and the increasing trend of $v_{2}$ from central to peripheral collisions is shown both in $p+\mathrm{Au}$ and $d+\mathrm{Au}$ collisions. This trend is due to a larger non-flow contributions in peripheral collisions. The AMPT simulation shows a larger $v_{2}\{\mathrm{EP}\}$ in $p+$ Au than that in $d+$ Au collisions, and this would be also related to the different non-flow contribution between the $p+\mathrm{Au}$ and $d+\mathrm{Au}$ collisions.

\section{Conclusion}

Collective-like behavior has been observed in small systems in PHENIX experiment. We measured $v_{2}$ and $v_{3}$ in $d+\mathrm{Au}$ and ${ }^{3} \mathrm{He}+\mathrm{Au}$ collision systems. Data are well described by viscous hydro models such as SONIC [6]. In addition to the system size dependence, we studied $v_{2}$ dependencies on collision energy, centrality, rapidity, and transverse momentum. The present study indicates that the non-flow contributions in $v_{2}$ could be important in small collision systems. This contribution needs additional study to fully understand the nature of the observed correlations in small systems.

\section{References}

[1] CMS Collaboration, J. High Energy Phys. 09, 091 (2010).

[2] PHENIX Collaboration, arXiv:1708.06983.

[3] S. Voloshin and Y. Zhang, Z. Phys. C 70, 665 (1996).

[4] PHENIX Collaboration, Phys. Rev. C 95, 034910 (2017).

[5] PHENIX Collaboration, Phys. Rev. Lett. 115, 142301 (2015).

[6] M. Habich, J. L. Nagle, and P. Romatschke, Eur. Phys. J. C 75, 15 (2015).

[7] Z.-W. Lin, C. M. Ko, B.-A. Li, B. Zhang, and S. Pal, Phys. Rev. C 72, 064901 (2005). 